Ingeniería Investigación y Tecnología, ISSN 2594 0732, I.3, 107-116, 2000

(artículo arbitrado)

DOI: http://dx.doi.org/10.22201/fi.25940732e.2000.01n3.012

\title{
Condiciones necesarias y acertijos matemáticos imposibles
}

\author{
M.A. Murray-Lasso \\ Unidad de Enseñanza Auxiliada por Computadora \\ Departamento de Ingeniería de Sistemas \\ División de Estudios de Posgrado, Facultad de Ingenieria, UNAM \\ Email: mamurrayservidor.unam.mx
}

(recibido: enero de 1999; aceptado: marzo de 1999)

Resumen

Este artículo se orienta principalmente a maestros de matemáticas ya que presenta varios acertijos denominados imposibles. Aquí se discuten las razones por las cuales dichos acertijos son imposibles de resolver. Además, se tratan instancias de acertijos que no cumplen con ciertas condiciones necesarias para que exista una solución. En algunos casos, es necesario introducir nuevos conceptos y herramientas para expresar estas condiciones necesarias, lo cual brinda al profesor la oportunidad de proporcionar a los estudiantes una motivación para estudiar dichos tópicos. El trabajo concluye demostrando la poca dificultad para crear nuevos acertijos imposibles tomando cualquier teorema de las matemáticas aún no visto en clase y así retar al alumno a que viole el teorema dentro del dominio de su validez.

Descriptores: acertijo; demostración; imposible; teorema; solución.

\section{Abstract}

This article is of particular interest to professors of mathematics because it presents several riddles that have been regarded as impossible. Reasons are discussed why such riddles are impossible to solve. Certain riddles that fail to comply with certain conditions necessary for a solution to exist are also dealt with. In some cases it becomes necessary to introduce new concepts and tools to express these necessary conditions, which in turn offers professors the opportunity of providing the students motivation for studying such topics. The work concludes by demonstrating the rather limited difficulty to create new impossible riddles taking into account any mathematical theorem even those not dealt with at school, therefore challenging the student to violate the theorem within its validity domain.

Keywords: riddle; demonstration; impossible; theorem; solution.

\section{Introducción}

Al demostrarse la aridez y el alto índice de reprobación que tienen en todos los niveles educativos las matemáticas, surge la necesidad de hacer que su aprendizaje sea más estimulante y si es posible, divertido. Entre las opciones para lograr esta meta se encuentran, la introducción de las matemáticas recreativas y la combinación de su enseñanza con el uso de la computadora, aprovechando de ésta su carácter interactivo y la posibilidad que ofrece de manejar voz, colores, imágenes, animación, vídeo y sonido. Este artículo estudia el tema relacionado con las matemáticas recreativas.

Se pueden introducir muchos conceptos matemáticos de forma amena a través de acertijos, magia matemática, juegos, etc. Existen numerosos acertijos matemáticos aparentemente sencillos, que en realidad, son imposibles de resolver. Algunos de ellos son: trazar una figura de líneas con un trazo continuo sin levantar el lápiz ni trazar ninguna de las líneas más de una vez; el "juego de 15" que consiste en un tablero con cuadrados deslizantes numerados del 1 al 15, más un hueco para que los cuadrados se puedan mover, 
DOI: http://dx.doi.org/10.22201/fi.25940732e.2000.01n3.012

pasar de una cierta configuración (por ejemplo, los números ordenados de manera creciente en filas consecutivas) a una segunda configuración (números en orden decreciente en filas); pasear un caballo de ajedrez usando movimientos legales y partiendo de un escaque específico, pasar por todos los escaques del tablero y terminar en un escaque específico.

En muchos casos, utilizando ideas sencillas, se puede demostrar que no es posible resolver los acertijos, aunque generalmente, es difícil que se le ocurra esa idea sencilla a un estudiante promedio. Este trabajo muestra algunos casos en que sí es posible lograr dichas demostraciones. La principal técnica que se utilizará será encontrar una condición necesaria para la solución que no cumple el caso específico que se quiere resolver. Algunos de los ejemplos que se enmarcan son famosos en la historia de las matemáticas y han originado nuevas ramas de la misma. Comenzaremos con uno que originó la teoría de los gráficos.

\section{Trazo de figuras de líneas sin levantar el lápiz ni duplicar segmentos}

La figura 1 no se puede dibujar sin levantar el lápiz ni trazar dos veces algún segmento. La siguiente demostración matemática afirma con toda certeza que para dibujar la figura con un trazo continuo, es necesario que en cada nodo (puntos donde se unen las líneas) exceptuando posiblemente dos de ellos, el número de líneas que se juntan en él sean par.

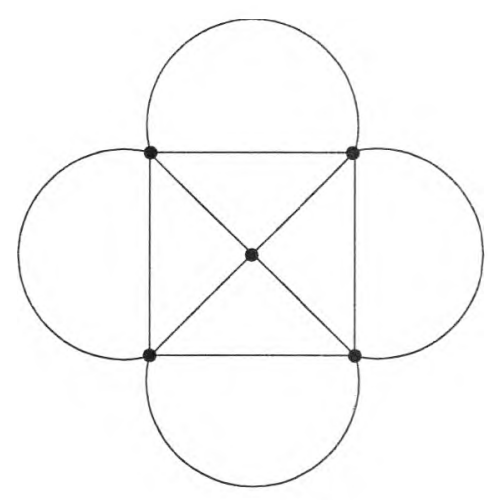

Figura 1

El siguiente argumento para demostrar la necesidad de la condición se debe a L. Euler, gran matemático suizo que fundó la teoría de gráficas (1736). Para que una figura sea delineada con un solo trazo a cada nodo intermedio, se debe llegar por una línea y salir por otra, por lo que el número de líneas incidentes con cada nodo intermedio deberá ser par. Solamente el nodo inicial y el nodo final, en caso de que sean diferentes, podrán tener un número impar de líneas incidentes, una por donde se sale del nodo inicial y la otra por la que se llega al nodo final, siendo un número par las líneas adicionales, si las hay, que utilizaron dichos nodos como nodos intermedios. Por iguales razones, si se desea dibujar la figura con un solo trazo y terminar en el mismo punto en el que se comienza, entonces todos los nodos deberán ser incidentes con un número par de líneas.

En vista de que en la figura 1 todos los nodos, excepto el del centro, tienen un número impar de líneas incidentes, la figura no cumple la condición necesaria y no se puede dibujar de un solo trazo. Si a la figura 1 se le eliminaran las diagonales del cuadrado del centro, entonces los cuatro nodos restantes tendrían cuatro líneas incidentes con cada uno y se cumpliría la condición necesaria, por lo que la figura modificada sí se podría dibujar con un solo trazo y se podría partir de cualquier nodo para terminar en el mismo. Si en cambio, nada más se elimina la línea que va de noroeste a sureste dentro del cuadrado central, entonces dos de los nodos (el inferior izquierdo y el superior derecho) tienen 5 líneas incidentes y los demás un número par de líneas incidentes. La figura sí se puede dibujar de un solo trazo, pero es necesario comenzar en uno de los nodos con 5 líneas incidentes y terminar en el otro nodo con 5 líneas incidentes.

Hay muchas variantes del acertijo presentado, en algunas hay que cruzar puentes una sola vez, en otras hay que pasar por ciertas puertas una sola vez, etc. En todos ellos se aplican las mismas reglas adaptadas a la situación, haciendo coincidir las puertas o puentes con las líneas de una gráfica.

\section{Números expresados como sumas de números naturales sucesivos}

Nótense las siguientes igualdades:

$$
\begin{aligned}
& 100=18+19+20+21+22, \\
& 101=50+51, \\
& 102=24+25+26+27, \\
& 103=51+52, \\
& 104=2+3+4+5+6+7+8+9+10+11+12+13+14, \\
& 105=52+53, \\
& 106=25+26+27+28, \\
& 107=53+54 \\
& 108=10+11+12+13+14+15+16+17, \\
& 109=54+55 \\
& 125=62+63 \\
& 126=10+11+12+13+14+15+16+17+18, \\
& 127=63+64 \\
& 128=? ? ? \\
& 129=64+65 \\
& 130=4+5+6+7+8+9+10+11+12+13+14+15
\end{aligned}
$$


Parecería que es posible expresar cualquier entero positivo entre 100 y 130 como la suma de varios números naturales sucesivos. Ciertamente, cualquier número impar se puede representar como la suma de dos números sucesivos. Si dicho número es $k$, entonces el número se puede escribir como $k=\operatorname{INT}(k / 2)+[\operatorname{INT}(k / 2)+1]$, donde la función INT aplicada a un argumento fraccionario da como resultado el entero más próximo a la izquierda del argumento, y aplicada a un entero resulta el mismo entero. Se reta al lector a que exprese 128 como la suma de varios números naturales sucesivos. Por más que lo intente no lo logrará. ¿Por qué?

Se deduce una propiedad de los números que pueden ser representados como la suma de dos o más números naturales sucesivos. Trabajando abstractamente, sea el primer número de la suma $k, n$ el número de términos, y $S$ la suma. Entonces se tiene

$$
\mathrm{S}=k+(k+1)+\ldots+(k+n-1)
$$

Para encontrar una expresión para esta suma, hacemos lo que hizo el gran matemático alemán K.F. Gauss para obtener la suma de los primeros $n$ números naturales: escribimos la suma dos veces, una de ellas ordenada a la inversa para obtener

$$
\begin{gathered}
S=k+(k+1)+\ldots+(k+n-1), \\
S=(k+n-1)+(k+n-2)+\ldots+k .
\end{gathered}
$$

Sumando los renglones y sacando mitad obtenemos

$$
\begin{aligned}
S=[2 k+n-1 & +2 k+n-1+\ldots+2 k+n-1] / 2 \\
& =n(2 k+n-1) / 2 .
\end{aligned}
$$

Existen dos posibilidades: 1) Que $n$ sea par y por ende $n / 2$ entero, en cuyo caso el factor entre paréntesis será impar y mayor que dos; y 2) que $n$ sea impar y la mitad del factor entre paréntesis entero, en cuyo caso el primer factor de $S$, es decir $n$, es impar - aunque parece que estamos asegurando que todo número natural expresado como una suma de números naturales sucesivos se puede factorizar, uno de los factores $n / 2$, podría valer la unidad sin violar las suposiciones, por lo que no se están excluyendn los números primos-. En ambos casos, uno de los factores de. número $S$ es impar y mayor que 2 ; en otras palabras, cual. quier número natural que se pueda expresar como una suma de $n \geq 2$ sumandos de números naturales tendrá un factor impar mayor que 2 . Nótese que para los números primos el factor impar es el número mismo y que todos los números primos excepto 2 son impares. Anteriormente se mostró una regla sencilla para expresar números impares (incluyendo los números primos impares) como la suma de dos números naturales sucesivos.

¿Cuáles números no tienen ningún factor impar mayor que 1? Solamente las potencias de 2. Por lo tanto, no se pueden expresar como sumas de números naturales los números $2,4,8,16,32,64,128, \ldots 2^{k}, \ldots$.

Hasta aquí sólo se ha demostrado que para que un número sea la suma de números naturales positivos es nece-

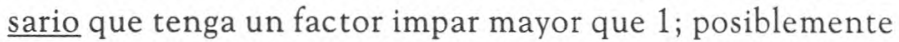
algún número diferente de las potencias de 2 no se pudiera expresar como una suma de números naturales sucesivos, es decir, quizá la condición no sea suficiente. Como ya se demostró que todos los números impares se pueden expresar como sumas de 2 números naturales sucesivos, restaría demostrar que cualquier número par que no sea una potencia de dos, también se puede expresar como una suma de números naturales sucesivos.

Sea un número par cualquiera $\mathrm{N}$ que no sea potencia de 2 por el teorema fundamental de la aritmética - aquel que dice que todo número natural se puede factorizar como el producto de números primos elevados a ciertas potenciasel número se puede factorizar en dos: el primero, $P$, es una potencia de 2 , y el segundo, un número $Q$ impar - producto de una o más potencias de números primos impares-. El número se puede expresar como una suma de números naturales por lo menos de dos formas; en la primera, si se descompone el número $Q$, por el método especificado arriba, en la suma de dos números sucesivos $\mathrm{q}_{1}$ y $\mathrm{q}_{2}$, entonces yuxtaponiendo $\mathrm{P}-1$ números naturales sucesivos después de $\mathrm{q}_{2}$ y anteponiendo $\mathrm{P}-1$ números naturales sucesivos descendentes antes de $\mathrm{q}_{1}$ se tendrá el número $\mathrm{N}$ descompuesto en una suma de números naturales como se muestra en la siguiente ecuación:

$$
\begin{gathered}
N=P Q=\left(q_{1}-P+1\right)+\left(q_{1}-P+2\right)+ \\
\left(q_{1}-1\right)+\underbrace{}_{1}+q_{2}+\left(q_{2}+1\right)+\ldots+\left(q_{2}+P-1\right)
\end{gathered}
$$

Nótese que cada uno de los pares unidos por líneas suman $\mathrm{q}_{1}+\mathrm{q}_{2}=\mathrm{Q}$ y que hay $\mathrm{P}$ pares. Para ejemplificar lo anterior considérese el número $2052=513 \times 4$. El número 513 se puede expresar como una suma de números naturales sucesivos como sigue

$$
513=256+257
$$

Poniendo 3 pares adyacentes a los anteriores (para completar los 4 requeridos) se tiene

$$
2052=253+254+255+256+257+258+259+260
$$

En algunos casos, es posible que algunos de los primeros números resulten negativos; dichos números se cancelarán 
con los números positivos a su derecha, ya que los números son sucesivos y los números centrales son positivos. Por ejemplo:

$$
24=8 \times 3 ; 3=1+2 ;
$$

$24=-6+-5+-4+-3+-2+-1+0+1+2+3+4+$
$5+6+7+8+9$

Cancelando los números negativos con los positivos e ignorando el cero se tiene

$$
24=7+8+9
$$

Una segunda manera de hacer la descomposición es la que se muestra en la siguiente ecuación

$$
\begin{gathered}
\mathrm{N}=\mathrm{PQ}=(\mathrm{P}-\mathrm{INT}(\mathrm{P} / 2))+(\mathrm{P}-\mathrm{INT}(\mathrm{P} / 2)+1)+\ldots+ \\
(\mathrm{P}-1)+\mathrm{P}+(\mathrm{P}+1)+(\mathrm{P}+2)+\ldots+(\mathrm{P}+\mathrm{INT}(\mathrm{P} / 2))
\end{gathered}
$$

en la cual se ha escrito $\mathrm{P}$ y se le ha rodeado de INT(P/2) números sucesivos de cada lado. Por ejemplo:

$$
112=7 \times 16=13+14+15+16+17+18+19
$$

También en este caso se pueden presentar números negativos a la izquierda, los cuales se cancelarán con números positivos a su derecha. Por ejemplo:

$$
\begin{aligned}
52=13 \times 4=-2+-1+ & 0+1+2+3+4+5+6+7+8 \\
& +9+10
\end{aligned}
$$

Cancelando los números negativos con positivos e ignorando el cero se tiene

$$
52=3+4+5+6+7+8+9+10 .
$$

Resumiendo lo anterior, se demostró que cualquier número impar puede expresarse como la suma de dos números naturales sucesivos. Que todos los números pares, que no sean potencias de 2 se pueden expresar como el producto de una potencia de 2 por un número impar mayor que 2, el cual, utilizando las técnicas mostradas aquí, se escribe como una suma de 2 o más números sucesivos. Los únicos números naturales que no se pueden escribir como sumas de números naturales sucesivos son las potencias de 2. Dichas potencias se van haciendo más raras al ir aumentando el exponente. Por ejemplo, entre 1 y 100 hay 6 potencias de 2 (que son: $2,4,8,16,32,64$ ); entre 100 y 200 solamente una (128); entre 200 y 300 existe una (256); entre 300 y 500 no hay ninguna; entre 500 y 1000 hay una (512); y entre 1000 y 2000 solamente una (1024).
Entonces, llama mucho la atención que todos los números entre 1000 y 2000 se puedan expresar como sumas de números naturales excepto 1024 .

Un acertijo interesante es mostrarle a un colega cómo se pueden expresar todos los números, excepto 64 , entre 50 y 100 como sumas de números naturales sucesivos y así retarlo a que haga lo mismo con el número 64. No lo podrá hacer, pero pensará que está a punto de lograrlo y lo seguirá intentando en forma similar como con los acertijos de dibujar figuras con un trazo continuo, las cuales no cumplen con la condición de Euler.

\section{Cubriendo pisos con mosaicos}

Supóngase que se tiene un piso cuadrado de dimensiones dos metros por dos metros, como se muestra en la figura 2 .

Se desea cubrir el piso con unos mosaicos rectangulares que miden 25 por $50 \mathrm{~cm}$. ¿Es posible hacerlo? La respuesta es muy fácil y es "sí". Una manera es: se colocan 8 filas de 4 mosaicos en cada una con las aristas más largas de los mosaicos en dirección horizontal.

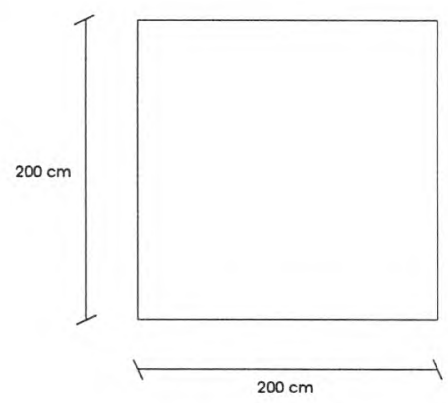

Figura 2

Ahora, resulta que en las esquinas superior izquierda e inferior derecha del cuarto, van a pasar unos tubos de sección cuadrada de $25 \mathrm{~cm}$ por lado. Se desea cubrir el piso con los mismos mosaicos rectangulares. Naturalmente que en la primera y octava fila ya no caben 4 mosaicos como en el caso anterior, pero sería posible colocar los mosaicos cruzados como se muestra parcialmente en la figura 3 , en la cual se han señalado los huecos cuadrados trazando sus diagonales.

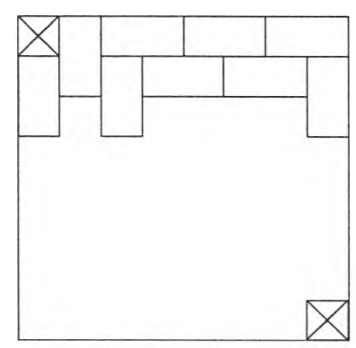

Figura 3 
Por más que se intente, no se podrán colocar los mosaicos rectangulares para cubrir el piso respetando los cuadrados por donde pasan los tubos. Los que lo intentan, siempre quedan con la impresión de que estaban a punto de lograrlo (análogamente a los acertijos de trazado continuo) y que solamente se requiere hacer un cambio ligero en las primeras decisiones para que se componga la parte final. La demostración de la imposibilidad de cubrir el piso con los mosaicos es muy simple, pero muy ingeniosa. Supongamos que el piso estuviera pintado como un tablero de ajedrez con cuadrados negros y blancos de $25 \mathrm{~cm}$ de lado y que un mosaico, equivalente a dos cuadrados de $25 \mathrm{~cm}$ de lado, puestos uno junto al otro, también tuviera pintado un cuadrado blanco y uno negro como se muestra en la figura 4.

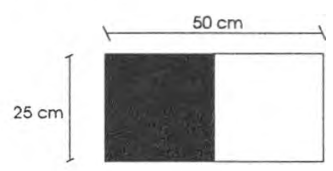

Figura 4

Obviamente las cosas no cambian porque el piso esté pintado como tablero de ajedrez, ni porque los mosaicos estén pintados como en la figura 4. Sin embargo, el imaginar que estén pintados proporciona una herramienta mental muy útil porque con ella podemos demostrar que no es posible cubrir el piso con los mosaicos rectangulares. La demostración va así: lo primero es notar que los dos cuadros cruzados de la figura 3 deben ser del mismo color, ya que en un tablero de ajedrez las diagonales están formadas con cuadros del mismo color. Por otra parte, al cubrir cualquier porción del piso con rectángulos como el de la figura 4, al terminar de colocar los mosaicos, el número de cuadros blancos y negros será el mismo, pero el espacio de piso a cubrir tiene dos cuadros menos del color de las esquinas cruzadas. Por lo tanto, pudo haber sido cubierto con mosaicos como el de la figura 4 .

\section{Posiciones imposibles en juegos de deslizamientos}

El gran acertijista norteamericano del siglo pasado Sam Loyd fue quien inventó el juego de 15; éste consiste en una caja cuadrada con 15 fichas y un hueco del mismo tamaño que se deslizan en dirección vertical y horizontal (figura 5). Una ficha adyacente al hueco puede intercambiar su posición con éste y por medio de una secuencia adecuada de deslizamientos, puede alcanzarse el objetivo del juego que es colocar las fichas en un determinado arreglo.

Por ejemplo, se tienen cuatro configuraciones o arreglos típicos (la $\mathrm{X}$ representa el hueco):

\begin{tabular}{|c|c|c|c|}
\hline 1234 & $\begin{array}{llll}1 & 2 & 3 & 4\end{array}$ & 15141312 & $\begin{array}{llll}15 & 9 & 13\end{array}$ \\
\hline $\begin{array}{llll}5 & 6 & 7 & 8\end{array}$ & 1213145 & $\begin{array}{llll}11 & 10 & 9 & 8\end{array}$ & $\begin{array}{llll}2 & 6 & 10 & 14\end{array}$ \\
\hline 9101112 & $11 \times 156$ & 765 & 371115 \\
\hline $\begin{array}{r}131415 X \\
\text { horizontal } \\
\text { (conf. Base) }\end{array}$ & $\begin{array}{c}10987 \\
\text { caracol }\end{array}$ & $\begin{array}{l}\begin{array}{llll}\begin{array}{lll}3 & 2 & 1\end{array} \\
\text { orden inverso } \\
\text { (imposible) }\end{array}\end{array}$ & $\begin{array}{l}4812 \mathrm{X} \\
\text { vertical }\end{array}$ \\
\hline
\end{tabular}

\begin{tabular}{|c|c|c|c|}
\hline 7 & 15 & 4 & 8 \\
\hline 9 & 5 & 12 & 14 \\
\hline 10 & 11 & & 6 \\
\hline 1 & 2 & 3 & 13 \\
\hline
\end{tabular}

Figura 5

Originalmente, el dispositivo se fabrica colocando las fichas en orden natural. Partiendo de dicho arreglo hay que hacer los deslizamientos necesarios para pasar a las diferentes configuraciones o arreglos.

Muchas de las configuraciones de este juego eran fáciles de obtener y algunos se volvieron muy diestros en el manejo del juguete. Había sin embargo, algunas configuraciones muy difíciles de lograr. Loyd ofreció una cantidad considerable de dinero al que lograra pasar de la primera configuración, de las ya demostradas, a la tercera (orden natural a orden inverso). Nadie pudo cobrar el premio. Algunos dijeron haber logrado la transición, pero olvidaban la secuencia seguida, lo que no les permitió repetir la proeza frente a los jueces. Otros presentaron la configuración lograda pero se descubrió que no la habían obtenido con deslizamientos, sino que habían desarmado el juguete y lo habían vuelto a armar. Desde la configuración imposible, por medio de deslizamientos, no pudieron pasar a la configuración base. Los que sabían mover la primera configuración no tenían dificultad con las configuraciones fáciles pues en lugar de poner las fichas en sus lugares en orden numérico, simplemente seguían la misma estrategia que la usada para la configuración base (orden natural) fijándose en los números que deberían quedar en cada fila.

Así pues, para lograr la configuración vertical comenzaban poniendo en la primera fila: $1,5,9$ y 13 ; luego, en la segunda colocaban los números $2,6,10,14$; en la tercera ponían $3,7,11,15$; en la cuarta: 4, 8, 12 y el hueco. Todo siguiendo la estrategia para lograr la configuración base. Sin embargo, al tratar de aplicar la misma estrategia para lograr la tercera configuración (orden inverso) siempre resultaba que, ya sea el 1 y el 2 , o el 3 y el 2 estaban inver. tidos, o alguna otra inversión aparecía y no era posible ponerlos a todos en orden inverso correcto.

La realidad de las cosas es que, como se verá a continuación, es imposible pasar de ciertas configuraciones 
a otras por medio de deslizamientos. Si se considera la primera configuración con el hueco en la esquina inferior derecha - al hueco lo podemos numerar como 16- como la configuración base, y la configuración final que se quiere lograr también tiene el hueco en la esquina derecha inferior, por medio de deslizamientos solamente podemos pasar a aquellas configuraciones que tienen la misma paridad de permutaciones.

Con esto se quiere decir que, al formar un arreglo lineal con los números tomados de izquierda a derecha y de arriba hacia abajo, línea por línea, los números de una configuración final tiene paridad impar si el número de intercambios de lugares adyacentes con respecto al orden naturalo básico del que se parte es impar, y tiene paridad par si dicho número de intercambios es par.

El concepto de paridad aparece también en el desarrollo de determinantes por la expansión de Laplace. Recordamos que un determinante de orden $n$ se calcula como la suma algebraica de componentes de un arreglo cuadrado de elementos con 2 índices, siendo el número de términos igual al número de permutaciones de los números $1,2, \ldots, n$ -donde $n$ es el orden del determinante- el cual resulta ser $n$ !. Para formar los términos, se colocan los primeros índices de los elementos en orden natural y como segundos índices se colocan todas las permutaciones posibles de los índices. Cada término tiene ya sea signo positivo o negativo. Tienen signo positivo los que tengan un número par de inversiones y negativo los que tengan un número impar de inversiones. Se cuenta una inversión por cada ocasión en que recorriendo los índices de izquierda a derecha aparezca un índice mayor que uno a su derecha. - El número de inversiones de una permutación resulta ser el mínimo número de veces que haya que intercambiar dos índices adyacentes para poner los números en el orden original (Muir, 1960) - . Para ilustrar la regla, tomemos un determinante de tercer orden. Los números en orden natural son 1, 2, 3. Con estos números podemos hacer las siguientes 6 permutaciones:

$$
\begin{aligned}
& 1,2,3 \\
& 1,3,2 \\
& 2,1,3 \\
& 2,3,1 \\
& 3,1,2 \\
& 3,2,1
\end{aligned}
$$

Poniendo los primeros índices en orden natural y los segundos índices de acuerdo con cada una de las permutaciones, se tienen los siguientes términos para un determinante de tercer orden:

$$
\text { 1. } \mathrm{x}_{11} \mathrm{x}_{22} \mathrm{x}_{33}
$$

$$
\begin{array}{llll}
\text { 2. } & x_{11} & x_{23} & x_{32} \\
\text { 3. } & x_{12} & x_{21} & x_{33} \\
\text { 4. } & x_{12} & x_{23} & x_{31} \\
\text { 5. } & x_{13} & x_{21} & x_{32} \\
\text { 6. } & x_{13} & x_{22} & x_{31}
\end{array}
$$

Para determinar los signos, se demuestra que en el primer término no hay que intercambiar nada (cero intercambios) para poner los segundos índices en orden natural. Por lo tanto, el término lleva signo +. En el segundo término, para poner los segundos índices en orden natural, hay que intercambiar el segundo con el tercero, por lo que siendo impar el número de intercambios, el término lleva signo -. En el tercer término, hay que intercambiar el primero con el segundo para poner los segundos índices en orden natural por lo que el término lleva signo-. En el cuarto término, hay que intercambiar el segundo con el tercero y luego el segundo con el primero para poner los segundos índices en orden natural, por lo tanto, el término lleva signo + . En el quinto termino, hay que intercambiar el primero con el segundo y luego el segundo con el tercero, por lo que el término lleva signo + . En el sexto término hay que intercambiar el primero con el segundo, después el segundo con el tercero y finalmente, el segundo con el primero, tres intercambios, por lo que el término tiene signo -. El determinante resulta ser entonces:

$$
\begin{aligned}
& x_{11} x_{22} x_{33}-x_{11} x_{23} x_{32}-x_{12} x_{21} x_{33}+ \\
& x_{12} x_{23} x_{31}+x_{13} x_{21} x_{32}-x_{13} x_{22} x_{31}
\end{aligned}
$$

Para revisar este trabajo podemos comparar el resultado con la conocida regla — que sólo funciona para determinantes de 3 orden-de poner debajo de las primeras tres filas las primeras dos filas repetidas y hacer el cálculo por diagonales, poniéndole signo + a las que yendo de arriba hacia abajo van de izquierda a derecha, y signo - a las que yendo de arriba hacia abajo van de derecha a izquierda. En el arreglo que a continuación se muestra, se marcan con flechas las diagonales mencionadas. Los signos coinciden aunque hay necesidad en algunos casos de tomar en consideración que el orden de los factores no altera el producto.

$$
\mathrm{X}_{21} \mathrm{X}_{22} \mathrm{X}_{23}
$$

Ahora que se ha hecho referencia a las permutaciones pares e impares, se pueden comparar las configuraciones primera y tercera del "juego de 15 " que se mostraron anteriormentes. Cada movimiento se logra intercambiando el 
hueco con alguna ficha, y el hueco se tiene que mover un número par de veces para terminar en el mismo lugar, ya que por cada unidad que se mueva en sentido horizontal hacia la derecha, en algún momento debe moverse la misma unidad hacia la izquierda; lo mismo sucede en el movimiento de abajo hacia arriba y de arriba hacia abajo. Para poder determinar la paridad de la configuración "orden inverso" recordemos algunas de las propiedades del grupo de las permutaciones (Carmichael,1956; Aleksandrov et al., 1963 y Smirnov, 1970).

Una permutación se puede representar encerrando entre paréntesis los índices en su orden natural en una línea y los índices permutados en una segunda, debajo de la primera. Por ejemplo, la permutación que corresponde al sexto término del determinante de tercer orden analizado arriba se puede representar con la notación mencionada con

$$
\left(\begin{array}{lll}
1 & 2 & 3 \\
3 & 2 & 1
\end{array}\right)
$$

La notación utilizada nos indica que el número 3 está ocupando la posición originalmente utilizada por el número 1; que el número 2 está ocupando la misma posición que el 2 original y que el número 1 ocupa la posición que originalmente tenía el número 3. La misma permutación se puede escribir sin que los números en el renglón superior tengan que aparecer en orden natural; lo importante es la correspondencia de los números de las dos líneas. Así, la misma permutación se puede escribir

$$
\left(\begin{array}{lll}
2 & 3 & 1 \\
2 & 1 & 3
\end{array}\right)
$$

Existe un conjunto de permutaciones llamadas cíclicas en las cuales, si se tienen en el renglón superior los elementos $\mathrm{x}_{1}, \mathrm{x}_{2}, \mathrm{x}_{3}, \ldots, \mathrm{x}_{\mathrm{n}}$ entonces en el renglón inferior se tienen respectivamente $x_{n}, x_{1}, x_{2}, x_{3}, \ldots, x_{n-1}$. Dado que está determinada la correspondencia, para representar una permutación cíclica basta especificar el primer renglón. Así, la permutación cíclica ( $\left.\begin{array}{lll}3 & 1 & 2\end{array}\right)$ corresponde a la permutación

$$
\left(\begin{array}{lll}
3 & 1 & 2 \\
2 & 3 & 1
\end{array}\right)
$$

Cualquier permutación no cíclica se puede escribir como el producto de dos o más permutaciones cíclicas. - Se interpreta el producto $\mathrm{P}_{2} \mathrm{P}_{1}$ de dos permutaciones como la aplicación de la permutación $\mathrm{P}_{1}$ seguida de la permutación $\mathrm{P}_{2}$. En general, el producto de dos permutaciones no conmuta porque no se obtiene el mismo resultado aplicándolas en diferente orden; sin embargo, las permutaciones cíclicas que no tienen elementos en común, sí conmutan. Esto lo debe verificar el lector poniéndose ejemplos específicos-. La descomposición de una permutación en el producto de permutaciones cíclicas lo ilustraremos con un ejemplo. Supóngase que se tiene la permutación

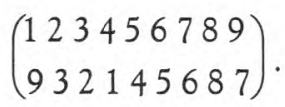

Comenzando con el primer símbolo en el renglón superior, se observa el símbolo en el inferior correspondiente, a continuación se consulta dicho símbolo en el renglón superior y se observa el símbolo correspondiente en el renglón inferior; se repite la acción anotando los símbolos en el orden en que son consultados en el primer renglón, hasta que se repita el primer símbolo. Eliminados dichos símbolos tanto del renglón superior como del inferior, se repite el proceso con los símbolos que quedan hasta que se agoten por completo. Aplicando el proceso descrito a la permutación mostrada arriba se anota (1976 9 4) y queda la permutación

$$
\left(\begin{array}{lll}
2 & 3 & 8 \\
3 & 2 & 8
\end{array}\right)
$$

la cual se descompone sucesivamente en (2 3) y (8). Podemos entonces escribir

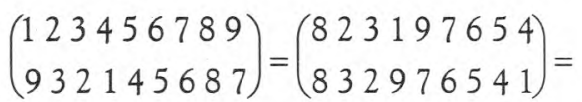

$$
\begin{aligned}
& \text { (8) }(23)(197654)=(197654)(23)(8)
\end{aligned}
$$

Lo que interesa ahora es contar el número de inversiones para saber si una permutación es par o impar. Se inicia por notar que las permutaciones cíclicas de un solo elemento no cambian dicho elemento, por lo que no tienen ninguna inversión, por lo tanto, se pueden ignorar en la cuenta. - De hecho, generalmente dichas permutaciones de un solo elemento ni siquiera se escriben, de la misma forma que se omite el coeficiente 1 frente a cualquier potencia de una variable en un polinomio-. A continuación notamos que

$$
\left(\mathrm{x}_{1} \mathrm{x}_{2} \mathrm{x}_{3} \ldots \mathrm{x}_{\mathrm{n}}\right)=\left(\mathrm{x}_{1} \mathrm{x}_{2}\right)\left(\mathrm{x}_{1} \mathrm{x}_{3}\right)\left(\mathrm{x}_{1} \mathrm{x}_{4}\right) \ldots\left(\mathrm{x}_{1} \mathrm{x}_{\mathrm{n}}\right)
$$

- Hay que recordar que debido a la arbitrariedad de la línea superior de la notación de doble línea para representar una permutación, los elementos $\mathrm{x}_{1}, \mathrm{x}_{2}, \ldots, \mathrm{x}_{\mathrm{n}}$ no necesariamente están en orden natural, por lo que $x_{1}$ no necesariamente está adyacente a $x_{2}$ cuando los elementos están en orden natural-. A cada permutación cíclica de 2 
elementos se le conoce como una transposición. Ahora, se citan tres teoremas de la teoría de permutaciones (Carmichael, 1956; Aleksandrov et al., 1963 y Smirnov, 1970).

Teorema 1 . Se puede obtener cualquier permutación de la permutación base por medio de transposiciones. - La demostración de este teorema se hace observando que cualquier permutación se puede descomponer en el producto de permutaciones cíclicas como se realizó anteriormente y que cualquier transformación cíclica se puede escribir como el producto de transposiciones, como también se demostró-.

Teorema 2. Debido a que existen expresiones como $(\mathrm{a} b)=$ $(\propto a)(\propto b)(\propto a)$, la manera de descomponer una permutación cíclica en transposiciones no está determinada, pero el número de ellas siempre es par o impar.

Teorema 3. Cada transposición cambia en un número impar el número de inversiones de una permutación.

Regresando al acertijo de Loyd de 15 fichas, notamos que si numeramos el hueco con 16, entonces lograr un cierto arreglo de las fichas se hace siempre por medio de transposiciones del tipo ( $16 n)$, donde $n$ es cualquier número de 1 a 15 . Si tanto la configuración inicial como final tienen al hueco ("ficha 16") en la misma posición, entonces necesariamente para poder llegar a la configuración final, ésta debe ser una permutación par de la configuración básica, pues al irse desplazando el hueco por las diferentes posiciones, como se señaló, tiene que bajar el mismo número de cuadros que antes subió y moverse hacia la derecha el mismo número de cuadros que antes se movió a la izquierda para regresar a su posición original. Por lo tanto, el número total de desplazamientos del hueco y el número total de transposiciones es un número par, por lo que la configuración final tiene que ser una configuración correspondiente a una permutación par. En el acertijo de 15 de Loyd existen 15 diferentes configuraciones, en las cuales el hueco está en la esquina inferior derecha. De esas configuraciones, la mitad son permutaciones pares y la mitad impares y no se pueden lograr las impares.

Vamos a analizar la configuración de orden inverso (imposible), la cual corresponde a la siguiente permutación

$$
\left(\begin{array}{l}
12345678910111213141516 \\
15141312111098765432116
\end{array}\right)
$$

$$
=\left(\begin{array}{l}
115 \\
\text { ) }
\end{array}(2 \text { 14) }(3 \text { 13) (4 12) (5 11) (6 10) (7 9) (8) }\right.
$$

Como se tienen 7 transposiciones, se trata de una permutación impar y por lo tanto, es imposible lograrla con un número par de deslizamientos. Por ello, está justificado que sea calificado como imposible. Si en vez de la configuración de orden inverso se intenta una configuración de orden inverso alternativa, como se muestra a continuación

$\begin{array}{rrrr}\mathrm{X} & 15 & 14 & 13 \\ 12 & 11 & 10 & 9 \\ 8 & 7 & 6 & 5 \\ 4 & 3 & 2 & 1\end{array}$

donde el hueco está en la esquina superior izquierda, entonces la permutación correspondiente es

$$
\begin{aligned}
& \left(\begin{array}{l}
12345678910111213141516 \\
16151413121110987654321
\end{array}\right) \\
& =(116)(215)(314)(413)(5 \text { 12) (6 11) (7 10) (8 9) }
\end{aligned}
$$

Como son 8 las transposiciones, se trata de una permutación par. Por otro lado, para desplazar el hueco de la esquina inferior derecha a la esquina superior izquierda se requiere un número par de transposiciones con el hueco, dando como resultado que la configuración sea posible, como lo puede verificar el lector si se compra o construye su "juego de 15," que por cierto en México lo venden en algunos mercados populares con el nombre de "Rompecabezas".

Finalmente, se analiza la configuración "caracol", la cual corresponde a la siguiente permutación

$$
\left(\begin{array}{l}
123456678910111213141516 \\
12341213145111615610987
\end{array}\right)
$$

$$
\begin{aligned}
= & (1)(2)(3)(4)(5126131016714911158) \\
= & (512)(56)(513)(510)(516)(57)(514)(59)(511) \\
& (515)(58)
\end{aligned}
$$

Se tienen 11 transposiciones, - lo cual no quiere decir que ése es el número de movimientos del hueco- por lo tanto es una permutación impar. Por otra parte, para llevar el hueco de la esquina inferior derecha a la posición de la X en la configuración del caracol por cualquier camino, se requiere un número impar de transposiciones, por lo que la configuración no es imposible en el sentido en que se ha estado discutiendo en este artículo.

No ha sido demostrado que las configuraciones que no son imposibles en el sentido en el que se ha estado tomando esta palabra en este trabajo, sean posibles. Eso se demostraría dando el algoritmo que lleve las fichas de una configuración base a la configuración deseada bajo la suposición que es de la paridad adecuada y demostrando la forma correcta de dicho algoritmo. El algoritmo existe y seguramente lo conoce cualquiera que haya jugado con el acertijo de 15, el cual no se discutirá aquí, ya que el enfoque del artículo es señalar algunos acertijos imposibles de resolver 
por haber condiciones necesarias para su solución que no se cumplen, razón por la cual, el que intenta resolverlo, se puede pasar una eternidad intentándolo sin lograrlo.

\section{Un acertijo imposible de geometría}

El teorema de Von Aubel (Gardner, 1981 y Kelly, 1966) es poco conocido por los que no son especialistas en Geometría. El teorema dice que si se tiene un cuadrilátero cualquiera y se construye un cuadrado sobre cada lado como se muestra en la figura 6 , y se unen con segmentos de línea recta los centros de los cuadrados correspondientes a los lados opuestos del cuadrilátero, los dos segmentos de recta son perpendiculares entre sí y de igual longitud. - Nótese que el teorema no asegura que los segmentos de recta se cruzan y menos que se bisecan-.

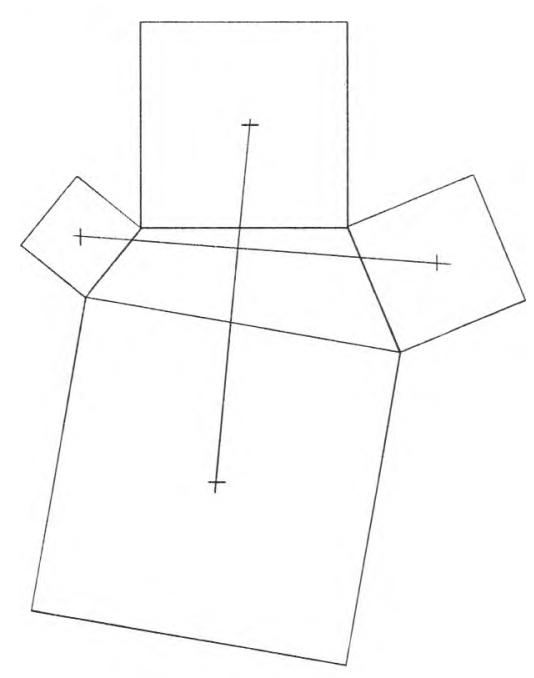

Figura 6

Un posible acertijo matemático que se podría plantear alrededor del teorema de Von Aubel es retar al lector a construir un cuadrilátero tal que las líneas que unen los centros de los cuadrados construidos sobre los lados, como se muestra en la figura 6, formen un ángulo mayor de 100 grados. De acuerdo al teorema de Von Aubel, no se puede construir dicho cuadrilátero. La demostración del teorema, que no damos por falta de espacio, admite cuadriláteros cuyos vértices caen arbitrariamente en el plano. De esta manera, aunque la figura 6 muestra un cuadrilátero convexo, se admiten cuadriláteros no convexos, cuadriláteros con sus cuatro vértices sobre una misma línea, cuadriláteros con algunos lados de longitud cero (por lo que los correspondientes cuadrados tendrán también lados con longitud cero)y cuadriláteros cuyos lados se cruzan; la única condición es que están cerrados. Para definir sobre cuál semiplano se colocan los cuadrados correspondientes a cada lado se define arbitrariamente un vértice inicial y un sentido de recorrido de los vértices; así, se coloca el cuadrado en el semiplano derecho con respecto a la dirección de recorrido. De esa forma, la figura 6 define como vértice inicial la esquina inferior derecha del cuadrilátero (punta más aguda) y el sentido de recorrido de los vértices como el contrario a las manecillas del reloj, los cuadrados mostrados aparecen a la derecha del recorrido. Sin embargo, se puede definir el sentido de recorrido opuesto, en cuyo caso los cuadrados se construirían hacia adentro del cuadrilátero. Aun en ese caso el teorema es válido. La generalidad del teorema hace verdaderamente imposible resolver el reto, a pesar de que el alumno intente recurrir a trucos. Desde el punto de vista educativo, es muy ilustrativo lo que significa un teorema que ha sido correctamente demostrado; es decir, no hay excepciones si se cumplen las reglas originales del juego dentro de las que se demostró el teorema.

\section{Conclusiones}

Hemos presentado algunos ejemplos de acertijos imposibles de resolver debido a que no se cumplen las condiciones necesarias para que tengan solución. Se tomaron varios casos que aparecen en libros de acertijos matemáticos, los cuales casi nunca explican por qué es imposible obtener soluciones, sino simplemente informan en la sección de respuestas que es imposible, aunque en algunos casos dejan al lector con la duda.

Muchos maestros consideran que los acertijos vuelven más motivante el aprendizaje de las matemáticas y por lo tanto retan a sus alumnos a resolverlos. Los más intrigantes son los sencillos de plantear y difíciles o imposibles de resolver, sobre todo, cuando aparentemente la solución debería ser sencilla. Esto presenta la oportunidad educativa de que los estudiantes intenten con gran intensidad la solución de un problema y por lo tanto aprecien en toda su dimensión la explicación posterior sobre cómo resolverlo o, en su caso, entender por qué no existe solución.

Cualquier teorema de las matemáticas se puede prestar al diseño de un acertijo. Cuando el teorema es muy conocido algunos estudiantes se darán cuenta inmediatamente de la imposibilidad de violarlo. Un ejemplo sencillo sería retar a los estudiantes a construir un triángulo cuyos ángulos internos sumen, digamos, 150 grados. El bajo nivel educativo de la población de nuestro país hace que dicho problema, exhibido como demasiado inocente, le imponga a ésta un verdadero reto; no se diga a personas sin educación secundaria, sino aun a muchos profesionales.

Teoremas menos conocidos, como el de Von Aubel ilustrado anteriormente, son verdaderos retos para los buenos estudiantes, por lo que difícilmente olvidarán la experiencia después de intentar verdaderamente resolver el 
DOI: http://dx.doi.org/10.22201/fi.25940732e.2000.01n3.012

acertijo y recibir una explicación, acompañada de una demostración matemática, de que se trata de un teorema.

Los estudiantes de matemáticas en general, aborrecen las demostraciones matemáticas y el estudio de temas a los que no les ven aplicación inmediata. La técnica de anteceder a la demostración de un teorema o a la introducción de nuevos conceptos un acertijo retante será muy útil para reducir dicho aborrecimiento.

En este trabajo se explican algunos conceptos de teoría de permutaciones. Dichos conceptos serían demasiado áridos, en general, si son introducidos sin previa motivación. No lo son si los alumnos llevan varios días intentando lograr una configuración final imposible en el "juego de 15" y se les explica que, dependiendo de si una permutación es par o impar, la solución es imposible o no, y se les promete usar los nuevos conceptos para analizar la configuración que tanto trabajo les está costando y demostrar que es imposible de lograr.

Otra técnica exitosa para motivar a los estudiantes es presentar el cumplimiento de un teorema como un acto de magia, cosa que también les gusta a los estudiantes. Así, el maestro puede decir que por medio de telepatía va a inducir a un estudiante a que dibuje un cuadrilátero tal, que al construir cuadrados sobre sus lados, los centros de los cuadrados de los lados opuestos queden a la misma distancia. A los estudiantes les gusta conocer los trucos de los magos, por lo que después de presentar "la magia" con varios cuadriláteros, estarán intrigados y querrán saber por qué funciona "el truco". En ese momento tendrán una disposición adecuada para que el maestro demuestre el teorema, convirtiendo una situación que normalmente es de rechazo en otra en la cual los estudiantes demanden saber por qué y si siempre funciona. iPocas veces logra el maestro crear una situación semejante en una clase de matemáticas!

\section{Referencias}

Aleksandrov A.D., Kolmogorov A.N. y Lavrent'ev M.A. (1963).Capítulo XX: Groups and other algebraic systems. Mathematics: Its Content, Methods, and Meaning, Vol. 3.

Carmichael R.D. (1956). Introduction to the theory of groups of finite order. Dover Publications, Inc., Nueva York.

Euler L. (1736). Solutio problematis ad geometriam situs pertinantis. Anales de la Academiae Petropolitanae, Vol. 8, $128-140$.

Gardner M. (1981). Mathematical circus, Vintage Books, Random House, Nueva York.

Kelly P.J. (1966). Von Aubel's quadrilateral theorem. Mathematics magazine, enero de 1966, 35 - 37.

Muir T. (1960) A treatise on the theory of determinants. Dover Publications, Inc., Nueva York.

Smirnov V.I. (1970). Linear algebra and group theory, (revisada, adaptada y editada por R.A. Silverman), Dover Publications, Inc., Nueva York.

\section{Semblanza del autor}

Marco Antonio Murray-Lasso. Realizó la licenciatura en ingeniería mecánica-eléctrica en la Facultad de Ingeniería de la UNAM. El Instituto de Tecnología de Massachussetts (MIT) le otorgó los grados de Maestro en Ciencias en Ingeniería Eléctrica y Doctor en Ciencias Cibernéticas. En México, ha laborado como investigador en el Instituto de Ingeniería y como profesor en la Facultad de Ingeniería, UNAM durante 36 años; en el extranjero, ha sido asesor de la NASA en diseño de circuitos por computadora para aplicaciones espaciales, investigador en los Laboratorios Bell, así como profesor de la Universidad Case Western Reserve y Newak College of Engineering, en los Estados Unidos. Además, ha ocupado diversos puestos en sociedades profesionales relativas a la ingeniería, tanto nacionales como internacionales. Es coautor de libros y artículos técnicos, memorias de conferencias y revistas de investigación y divulgación. Actualmente, es jefe de la Unidad de Enseñanza Auxiliada por Computadora de la División de Estudios de Posgrado de la Facultad de Ingeniería, UNAM, investigador nacional y consultor de la UNESCO. 